\title{
ROAD TRAFFIC CONGESTION MANAGEMENT BASED ON A SEARCH-ALLOCATION APPROACH
}

\author{
Jamal Raiyn \\ Computer Science Department \\ Al Qasemi Academic College \\ Baqa El Gharbia, Israel \\ raiyn@qsm.ac.il
}

\begin{abstract}
This paper introduces a new scheme for road traffic management in smart cities, aimed at reducing road traffic congestion. The scheme is based on a combination of searching, updating, and allocation techniques (SUA). An SUA approach is proposed to reduce the processing time for forecasting the conditions of all road sections in real-time, which is typically considerable and complex. It searches for the shortest route based on historical observations, then computes travel time forecasts based on vehicular location in real-time. Using updated information, which includes travel time forecasts and accident forecasts, the vehicle is allocated the appropriate section. The novelty of the SUA scheme lies in its updating of vehicles in every time to reduce traffic congestion. Furthermore, the SUA approach supports autonomy and management by self-regulation, which recommends its use in smart cities that support internet of things (IoT) technologies.
\end{abstract}

Keywords: ITS, forecast scheme, road traffic management, location detection

\section{Introduction}

Various short-term forecasting schemes have been introduced to manage road traffic networks, reviewed in Raiyn and Toledo (2012). In general, the schemes that have been proposed involve forms of centralized management, which may achieve near- optimum performance in whole systems in regard to maximum capacity. However, as the number of vehicles increases, centralized computation may become mathematically intractable. Such schemes are also impractical when traffic varies significantly and creates difficulties for measuring actual conditions. The major disadvantage of centralized management is the occurrence of deadlock, which causes the whole system to collapse. We conclude that it is impractical to implement centralized control to handle Big Data, because it requires system-wide information, and the complexity of searching all possible reallocations is computationally hard. To reduce the overload in computational management time, we consider the management of urban road traffic in distributed form, and for this we propose the use of decentralized management. A decentralized algorithm would not use a central controller: rather, each process would be equivalent, cooperating with its neighbours to achieve a common end. The proposed approach is a combination of searching, updating and allocation (SUA) techniques; it aims to reduce several types of traffic congestion and their effects, such as delays, waiting time, driver stress, air and noise pollution, and economic costs (Kammoun et al., 2014). The search phase is used to determine the shortest route from the source to the destination node based on short- term forecasting. The forecasting model is based on the exponential moving average (EMA). The update phase is used to inform the system of new events during the trip, and the updated information is used to reduce road traffic congestion. In keeping with the updated information, vehicles are allocated the appropriate sections, and drivers can select new sections with low traffic congestion. In general, the SUA approach is characterized by the use of management by self-regulation. Furthermore, it offers several advantageous features, like autonomy and negotiation. Autonomy increases flexibility in dealing with new situations in the traffic load, while negotiation is used to avoid traffic congestion. This paper is organized as follows. In section 2, we describe the system. In section 3, we introduce the methodology of the SUA scheme, and in section 4, we provide an evaluation of the SUA approach and the simulation results.

\section{System Description}

We graph $G=(V, E)$ to model the road networks. $V$ is a set of nodes (vertices), and $E$ is a set of possible interconnections between pairs of nodes (edges). We assume that an agent manages the node. Nodes can communicate to solve problems involving heavy traffic loads over the arcs (sections). The 
proposed method considers the capacity in a section between two nodes, and the weight of the section. Each section aims to maximize capacity by distributing the heavy traffic load over all of the sections. Agents compute forecasts based on historical data $\left(E_{1}^{H}\right)$, real-time data $\left(E_{1}^{R}\right)$ and accidents ( $\left.E_{1}^{A c c}\right)$. To meet the challenge of the rapid growth of the internet of things (IoT), which promotes the growth of data, the system creates a new agent to manage newly received innovative data, which are received from new sources and unstructured data, which call for more analysis.

\section{Methodology}

\subsection{Travel Data Generation}

Travel data are collected from various sources. In this section, we mention four major strategies for collection: on-road sensors, video cameras, cellular phones, and GPSs. Currently, the most widely used, traditional strategy employs magnetic loop detectors, installed under the roadway surface (Chrobok et al., 2004). The scarcity of this technology is explained by the high cost of installing and maintaining the local detectors; they are typically installed in only a relatively small area of the roadway system, thus providing limited coverage of the entire transportation network (Raiyn, 2016). To avoid the cost of roadside equipment, we use cellular phone service to collect traffic data. Information based on cellular systems can be gathered in milliseconds compared to traffic data collected from detectors. Conceptually, these traffic data may fall into one of three categories: historical information, real-time information, and predictive information (Lee et al., 2008; Xia, 2010).

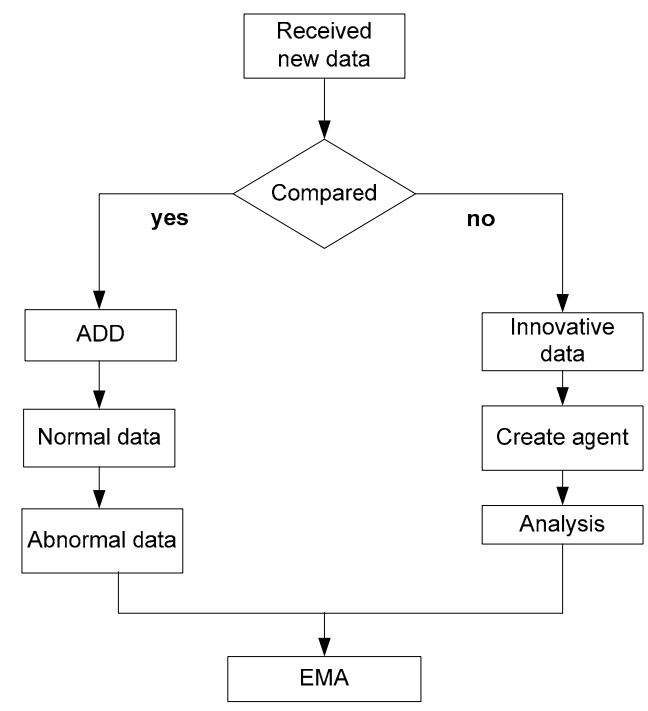

Figure 1. Innovative data

\subsection{Travel Big Data Management}

To reduce the processing time and the overload management, the forecasting is performed in distributed form. The forecast scheme combines three kinds of data; historical data, real-time data, and accident data.

\subsubsection{Short-Term Forecasting Model}

Various short-term traffic forecasting schemes have been proposed (Zhang and Rice, 2003; Tu et al., 2008; Zheng and Liu, 2009). In this section, we introduce a forecasting model based on the moving average. There are three types of moving averages: the simple moving average (SMA), the weighted moving average (WMA), and the exponential moving average (EMA). In this study, the exponential moving average is used.

The agent used in the EMA scheme analyzes the collected data in various ways. The accuracy of this technique depends on the weight smoothed alpha factor value and the gamma factor value of the current demand. To determine the optimal alpha factor value, we use a fitting curve. There are two major kinds of EMA forecasting. One uses EMA based historical information (HI); the other uses EMA based 
real-time information (RI). We add a new kind of EMA that focuses on innovative observation data, which consists of normal and abnormal (accident) travel data.

$t t_{\text {totally }}(k, t)=t t_{\text {normal }}(t, k)+t t_{\text {abnormal }}(t, k)+t t_{\text {innovative }}$.

\subsubsection{Forecasting Based on HI}

The historical database is a collection of past travel observations of the system. The exponential smoothing forecasting method assigns weight unequally to the observed time series. This is accomplished by using one or more smoothing parameters, which determine how much weight is given to each observation. The major advantage of the exponential smoothing method is that it gives good forecasts for a wide variety of applications. In addition, the data storage and computing requirements are minimal, which makes exponential smoothing suitable for real-time forecasting.

$t t(t+1, k)=\alpha * t t^{M}(t, k)+(1-\alpha) * t t^{H}(t, k)$

where $0<\alpha \leq 1, \mathrm{tt}^{\mathrm{M}}(\mathrm{t}, \mathrm{k})$ is the actual travel time in section $k$ at time $t$, and $\mathrm{tt}^{\mathrm{H}}(\mathrm{t}, \mathrm{k})$ is the historical travel time in section $k$ at time $t$.

\subsubsection{Forecasting in RI}

The occurrence of abnormal conditions in traffic flow information decreases the reliability of forecasts based on historical information and may increase the complexity of the forecasting of unusual incidents. The forecasting model based on real-time information gives little weight to historical information but great weight to real-time observations.

$t t(t+1, k)=t t^{H}(t+1, k)+\gamma *\left(t t^{M}(t, k)-t t^{H}(t, k)\right)$,

where $0<\gamma<1$.

\subsubsection{Forecasting in accident situations}

The informed iterative algorithm detects road accidents based on variation in travel time. We consider accidents during peak periods (i.e., morning or afternoon) and during non-peak periods. The observed traffic data consists of normal and abnormal (accident) travel data. The abnormal record consists of conditions involving traffic speeds that are at least $30 \mathrm{~km} / \mathrm{h}$ lower than the average speed of all the records for the same time on the same day of the week. The threshold of $30 \mathrm{~km} / \mathrm{h}$ is a symbolic value for the smallest speed change that people would consider "abnormal". The determination of this threshold depends on the travel observation data. Equation 4 is used to forecast the accident scheme.

$t t(t+1, k$, accident $)=\alpha * t t(t, k$, accident $)+(1-\alpha) * E M A(t, k$, accident $)$.

Alpha can be expressed as follows $\alpha=\frac{1}{1+\left[\frac{\operatorname{Var}(k)}{E(k)}\right]}$,

where, $E(k)$ is the expected number of crashes at the reference sites, and $\operatorname{Var}(k)$ is the variance in the expected number of crashes at the reference sites.

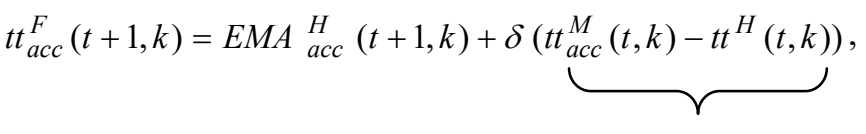

$$
\begin{aligned}
& \text { Corrections needed }=\text { epsilon }
\end{aligned}
$$

where $0<\alpha \leq 1,0<\delta<1, t t^{M}(t, k)$ is the actual travel time in section $k$ at time $t$, and $t t^{H}(t, k)$ is the historical travel time in section $k$ at time $t$. 


\subsubsection{Forecasting for unstructured data}

We add a new kind of EMA forecasting focused on innovative data.

$E M A_{\text {innovative }}(t+1, k)=E M A(t+1, k)+\gamma^{*}\left(\frac{t t^{M}(t, k)-E M A^{H}(t, k)}{\delta_{k}}\right)$,

where $\delta_{k}$ is the standard deviation for section $k$.

\subsection{Vehicle location}

A great deal of road equipment is used to determine and to track vehicle location. The most common devices are global positioning systems (GPSs), GNSSs, mobile phones, and other wireless networks. The generated information is used to update the SUA scheme and improve traffic flow management.

\subsection{The searching, updating and allocation approach (SUA)}

The processing time required for forecasting scheme for all the sections in real-time is lengthy and complex. To reduce the processing time, we propose a new approach based on searching, updating and allocation techniques (SUA). In the first phase, the approach searches for the shortest route based on historical observations computes the travel time forecast based on the vehicle's location, and updates the driver. This update is carried out for every period of time before section allocation. In the first phase, the SUA approach estimates the traffic flow in each section of the designated road network based on the EMA, which forecasts traffic flow in real time and under abnormal conditions (Raiyn, 2016). In the second phase, the SUA approach finds all $k$ routes from the source's starting point (start_point $S$ ) to the destination (end_point $D$ ) and selects the shortest route between them based on the EMA forecast.

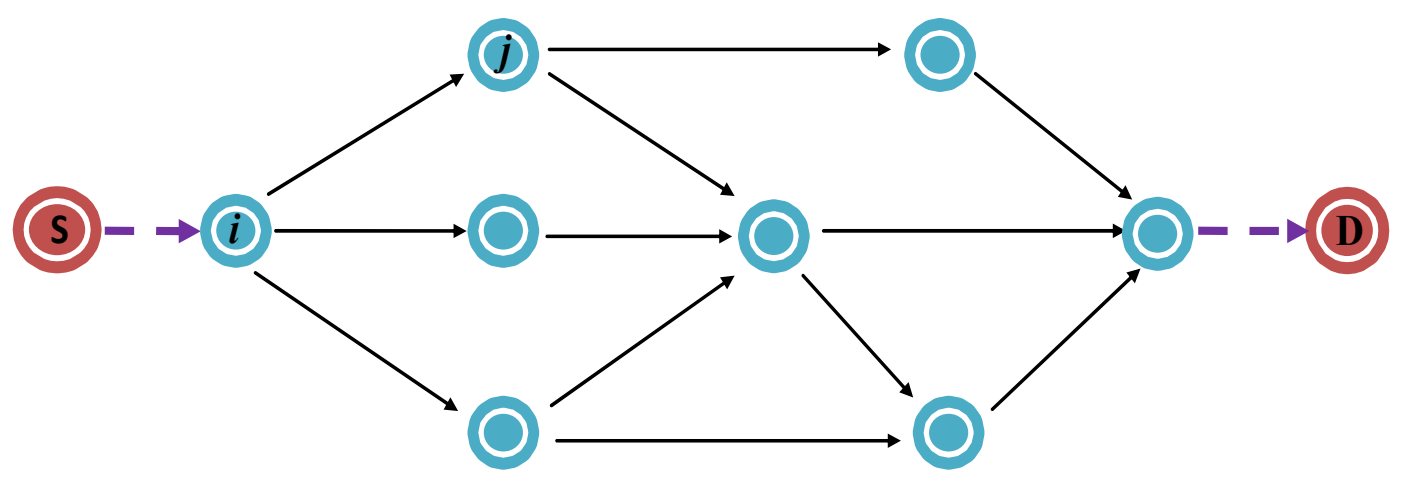

Figure 2. Road network

The SUA approach combines three modern techniques for urban road traffic management in smart cities.

\section{- Searching}

The nodes use two kinds of messages to communicate with their neighbouring nodes.

REQ (Req, node_name, ts, location), and RESP (Use, Free).

\section{- Updating}

Each section in the road network updates its status according to the capacity of the sections. Each node in the system uses the following local variables.

$\mathbf{U s e}_{\mathbf{i}}(\mathbf{k}$, time), node $i$ reports a heavy traffic load in section $k$ at time $t$.

Free $_{\mathbf{i}}(\mathbf{k}$, time), node $i$ reports light traffic load in section $k$ at time $t$.

A node $i$ keeps information about the status of the neighbouring nodes.

$$
\text { Available }(i, t)= \begin{cases}1 & \text { if } \operatorname{EMA}(\mathrm{k}, \mathrm{t})>\text { Speed }_{\text {threshold }} \\ 0 & \text { otherwise }\end{cases}
$$


Available $(i, t)$ is an array that keeps the available sections from the starting node (start_node $i$ ) at time $t$ until the end point (end_point). A node $i$ keep track of vehicles using the neighbouring sections. $H S_{i}$ is an array maintained by node $i$ that denotes the number of sections under a heavy traffic load.

\section{- Allocation}

Node $i$ communicates with neighbouring nodes, $N_{i}$. This communication between nodes is based on a message exchange protocol. Based on information received from neighbouring nodes, the protocol decides which section to allocate.

\subsection{Algorithm Description}

The goal of the SUA algorithm is to track the locations of vehicles, search for road sections with light traffic flow, and allocate them. Figure 3 illustrates the message exchange protocol between two nodes. Figure 4 diagrams the sequence of message exchanges.

Figure 5 describes the search phase of the SUA approach, which searches for sections with light traffic by sending request messages to neighbouring nodes. When a node receives a request message, it updates its local information as described in Figure 6, and allocates sections with light traffic as described in Figure 7.

\begin{tabular}{|c|c|c|c|}
\hline Node i & -Request & -Response & Node j \\
\hline -node_name: string & \multirow{6}{*}{ * } & \multirow{6}{*}{ * } & -NODE_NAME : string \\
\hline $\begin{array}{l}\text { +start_search() } \\
\text { +sendRequest() } \\
\text { +exit_search() }\end{array}$ & & & $\begin{array}{l}\text { +receivedMessage() } \\
\text { +sendResponse() } \\
\text { +update_status() }\end{array}$ \\
\hline$\Delta$ & & & $\triangle$ \\
\hline sendRequest & & & sendResponse \\
\hline $\begin{array}{l}\text {-req_type : string } \\
\text {-node_name : char } \\
\text {-ts : string }\end{array}$ & & & $\begin{array}{l}- \text { REQ_TYPE : string } \\
\text {-NODE_NAME : string } \\
\text {-TS : string }\end{array}$ \\
\hline & & & $\begin{array}{l}+ \text { Use }() \\
+ \text { +Grant }() \\
+ \text { Free() }\end{array}$ \\
\hline
\end{tabular}

Figure 3. Searching and updating process

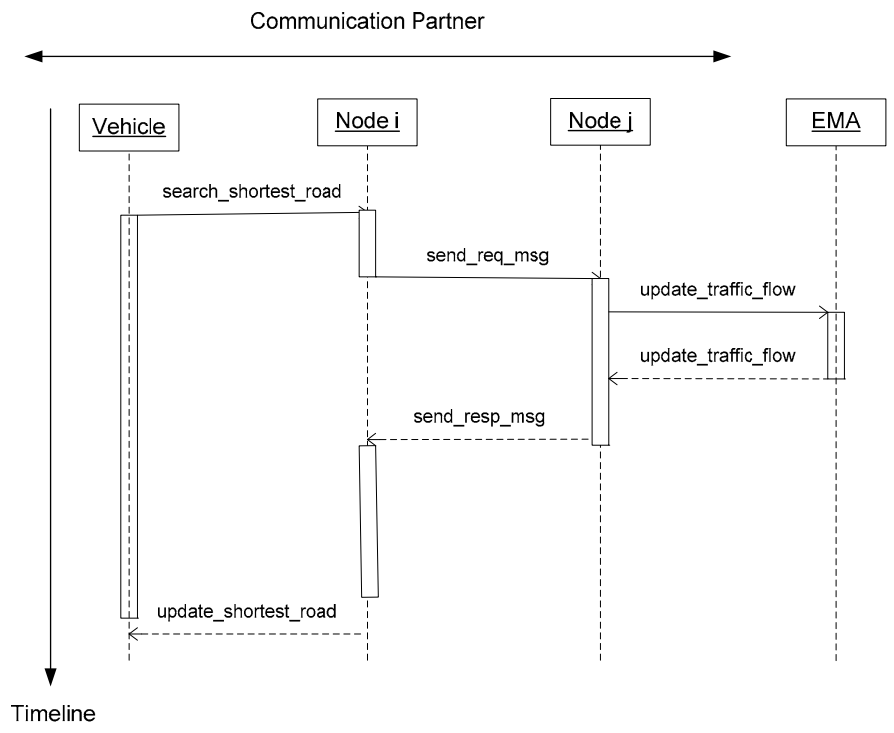

Figure 4. Sequence diagram for message exchange 


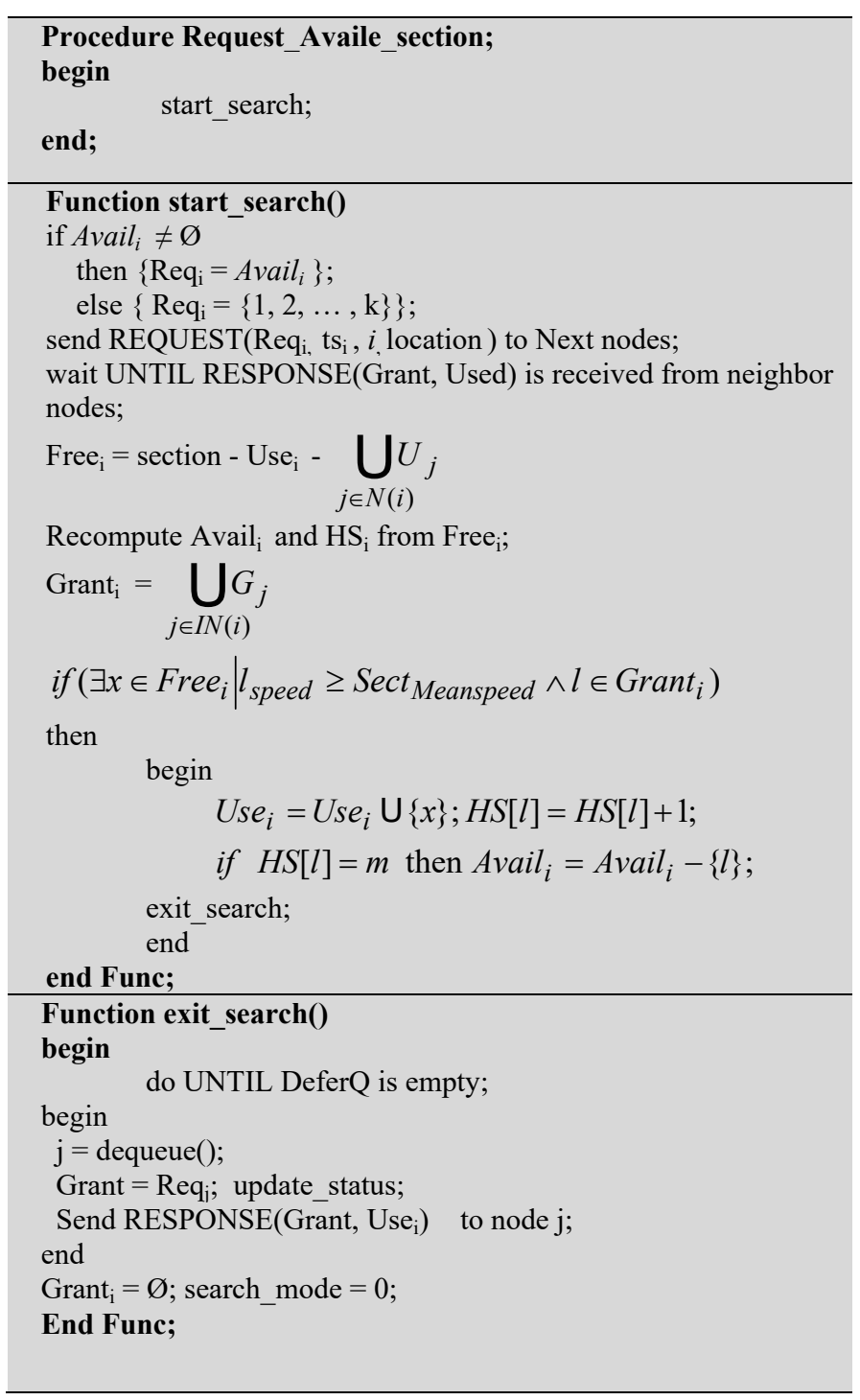

Figure 5. Node $i$ search section

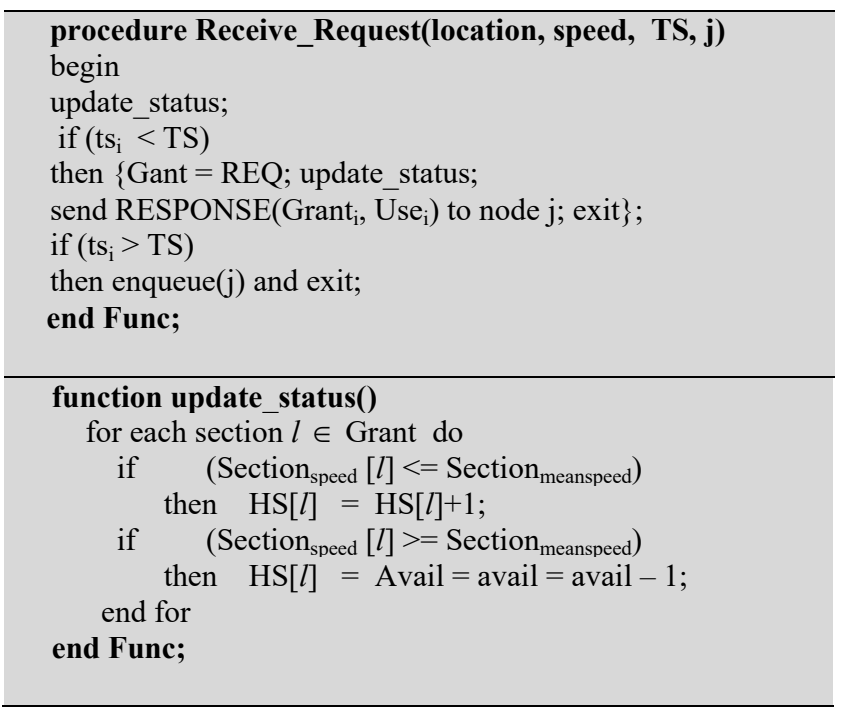

Figure 6. Node i receives request message from node $\mathrm{j}$ 


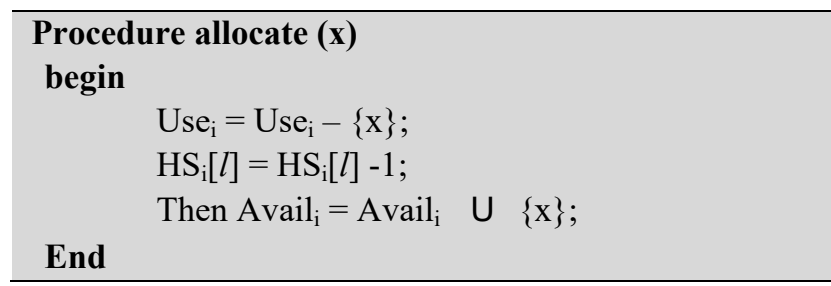

Figure 7. Node i allocates section $\mathrm{x}$

\section{Evaluation of the SUA Scheme}

We have analyzed the SUA scheme. The approach is deadlock free. A node cannot wait indefinitely, because the negotiation between two nodes is controlled and managed by the SUA system. When a node needs to allocate a new section, the SUA scheme informs about the traffic flow state in its neighbouring nodes. Then the node contacts a neighbouring node with light traffic flow. In this process, every node sends a response message in reaction to a request message it has received. Hence, in finite time (defined time), a given node receives all the response messages and either it is successful in acquiring a section or not. However when all of the nodes are involved in cyclic waiting a deadlock occurs. To prevent this, the argument can be extended to indicate that there should be no cyclic waiting regardless of the number of nodes. Consequently, deadlock cannot occur, because in the process of section allocation the nodes do not have priority over the sections in the neighbouring nodes. The first node that sends a request to acquire a section is the first node that can use that section.

\subsection{Simulation results}

The newly received data set is compared to the available data set. Innovative data are characterized by statistical measurement as illustrated in Figure 8. Figure 9 consists of three parts, each of which refers to a certain section of urban road. As illustrated in first part (A), the SUA scheme detects new data. Figure 10 consists of three parts as well: the third part illustrates the data processing time for the whole road network, which consists of 20 sections. The EMA scheme uses decentralized management which is based on a distributed arrangement, and it requires an average of 3.0576 seconds to process all of the road network information. For one section, however, only an average of 0.6240 of a second is required.

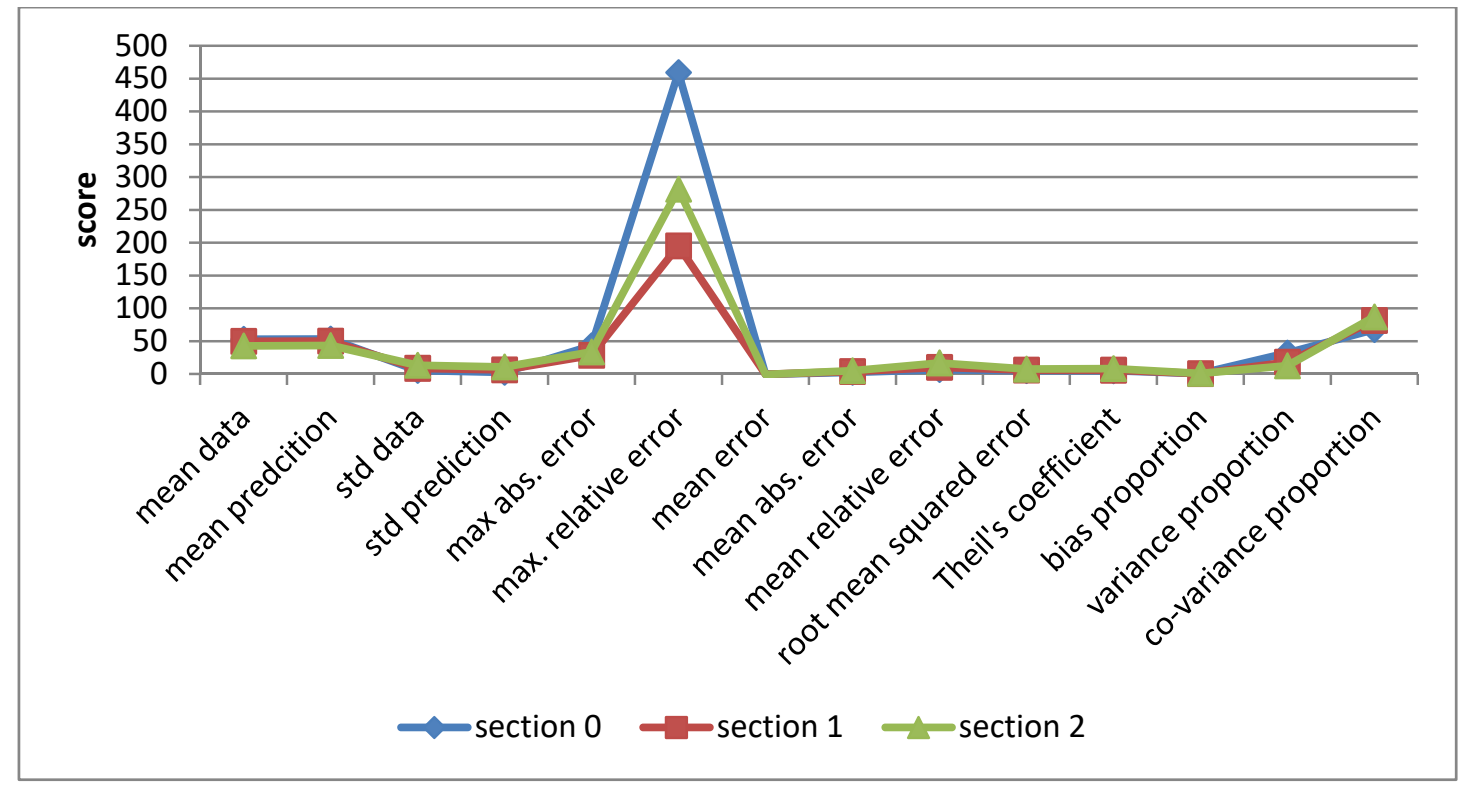

Figure 8. Data analysis based on statistical error measurement 

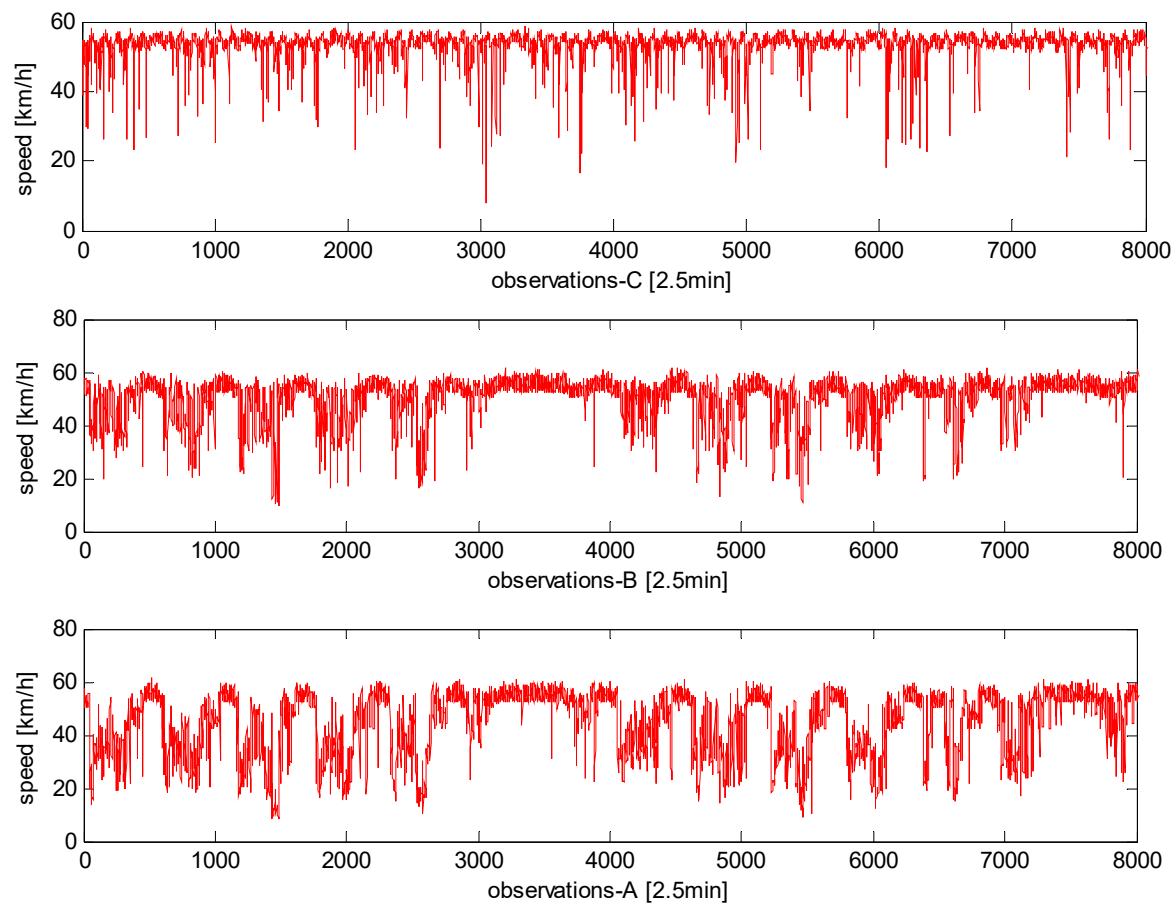

Figure 9. Innovative data detection
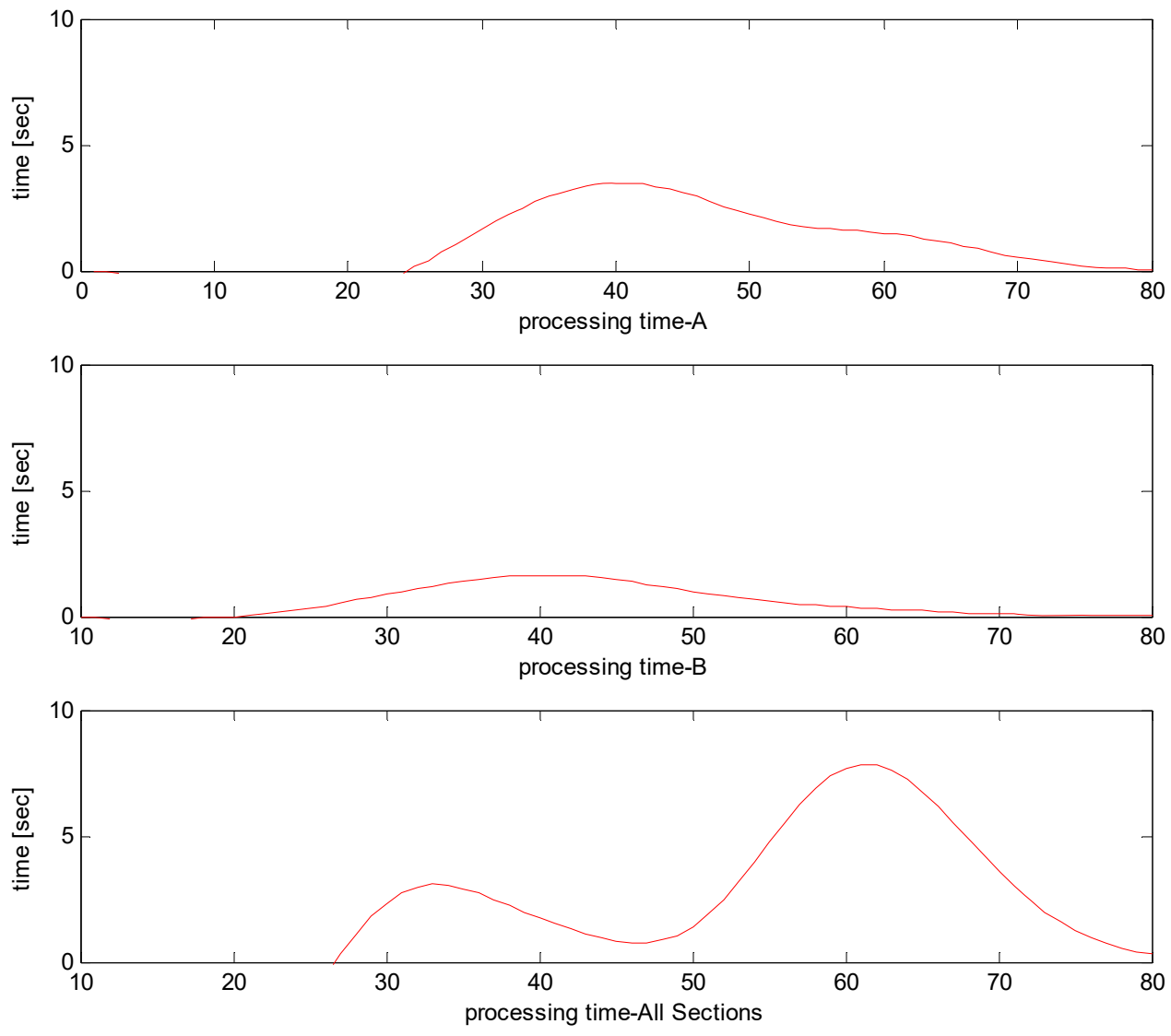

Figure 10. Processing time for data 


\section{Conclusion}

The centralized management of Big Data to assess traffic flow may become mathematically intractable. This method is also impractical under highly varying traffic loads because of the difficulty of measuring actual conditions. We conclude that centralized control is impractical because it requires system-wide information and the complexity of searching for all possible reallocations is computationally hard.

This paper introduces a new kind of road traffic congestion management that is based on decentralized control. The data processing time increases as the number of vehicles increases in the road networks, and as traffic congestion management becomes more complex. To reduce the data processing time, we propose the SUA approach, which uses message exchanging to establish a communication protocol between vehicles. The SUA approach has three phases: searching for the shortest path, updating the vehicle on the state of the roadway, and allocating the vehicle to an available road section.

The management time for road traffic congestion whole urban road networks is very high, and the SUA approach greatly reduces data processing time.

\section{Acknowledgement}

The author wishes to thank the Institute for Innovative Transportation at the University of Tel Aviv for funding this research.

\section{References}

1. Chrobok, R., Kaumann, O., Wahle, J. and Schreckenberg, M. (2004) Different methods of traffic forecast based on real data, European Journal of Operational Research, 15:558-568.

2. Lee, H., Chowdhury, K.N. and Chang, J. (2008) A New Travel Time Prediction Method for Intelligent, Transportation Systems. Springer-Verlag, Berlin, 2008. 473-483.

3. Lv, Y. and Tang, S. (2010) Real-time Highway Traffic Accident Prediction Based on the K-Nearest Neighbor Method. International Conference on Measuring Technology and Mechatronics Automation, 3: 547-550.

4. Kammoun, H.M., Kallel, I., Casillas, J., Abraham, A. and Alimi, M.A. (2014) Adapt-Traf: An adaptive multiagent road traffic management system based on hybrid ant-hierarchical fuzzy model, Transportation Research Part C 42 (2014) 147-167.

5. Karim, A. and Adeli, H. (2001) Fast Automatic Incident Detection on Urban and Rural Freeways Using Wavelet Energy Algorithm. Journal of Transportation Engineering, 2001; 129: 57-68.

6. Raiyn, J. and Toledo, T. (2012) Performance analysis and evaluation of short-term travel forecast schemes based on cellular mobile services. International Review of Civil Engineering. 3, 2, 201-205.

7. Raiyn, J. (2016) Speed Adaptation in Urban Road Network Management, Transport and Telecommunication, 17, 2. 111-121.

8. Tu, H., Van Lint, H. and Van Zuylen, H. (2008) The Effects of Traffic Accidents on Travel Time Reliability”, IEEE Conference on Intelligent Transportation Systems, Beijing, China, Oct; 12-15.

9. Xia, J. (2010) Predicting Freeway Travel Time under Incident Condition. Proceedings of Transportation Research Board's 89th Annual Meeting CD-ROM, Jan; 10-14; Washington, DC.

10. Xiaoqiang, Z., Ruimin, L. and Xinxin, Y. (2010). Incident Duration Model on Urban Freeways Based on Classification and Regression Tree. 2nd International Conference on Intelligent Computation Technology and Automation, TRB Annual Meeting, 2, 526-528.

11. Zhang, X. and Rice, J.A. (2003) Short-term travel time prediction. Transport. Res. Part C: Emer. Technol.; 11(3-4): 187-210.

12. Zheng, X. and Liu, M. (2009). An overview of accident forecasting methodologies, Journal of Loss Prevention in the Process Industries 2009; 22: 484-491. 